Cannon and colleagues developed an enzymelinked immunosorbent assay (ELISA) to measure serum levels of the corresponding protein, JM-27.

The authors obtained sera from 29 men with histologically confirmed, asymptomatic BPH and American Urological Association (AUA) symptom score $\leq 15,39$ men with symptomatic $\mathrm{BPH}$ and AUA score 16-32, and 17 men with confirmed prostate cancer and AUA score $<8$ (many samples were from participants in the Medical Therapy of Prostatic Symptoms Trial). JM-27 levels correlated with AUA scores, and were markedly different in men with highly symptomatic and asymptomatic $\mathrm{BPH}$, respectively. The sensitivity and specificity of the authors' ELISA in discriminating between high and low AUA scores were $90 \%$ and $77 \%$, and its positive and negative predictive values were $74 \%$ and $91 \%$ respectively. These findings were unaffected by prostate volume or the presence of prostate cancer. Intriguingly, JM-27 levels are reduced in sera from men with highly symptomatic $\mathrm{BPH}$, despite markedly increased expression of JM27.

Cannon and colleagues speculate that JM-27 levels could identify men with severe BPH before symptom onset, which might allow early use of aggressive therapy before irreversible bladder changes occur.

Original article Cannon GW et al. (2007) A preliminary study of JM-27: a serum marker that can specifically identify men with symptomatic benign prostatic hyperplasia. J Urol 177: 610-614

\section{Only trained and experienced medical personnel should perform circumcisions}

Around $25 \%$ of men worldwide are circumcised, mostly during childhood. In developed countries circumcisions are usually performed by medically qualified individuals, but many-especially those performed in religious ceremonies-are still done by untrained personnel, often without anesthesia. Complications occur in 1-15\% of cases and are usually minor, but severe complications-including glandular necrosis and amputations-also occur.

Ceylan and colleagues retrospectively reviewed medical records from 48 patients (aged 5 months to 24 years) with severe complications of circumcision that required surgical repair. The interval between circumcision and repair ranged from $2 \mathrm{~h}$ to 18 years. Only three patients had been circumcised in a medical center. Preputioglandular fusion was the commonest complication $(n=25)$; adhered areas were excised, the glans was sutured and the excess preputial tissue removed. Meatal stenosis $(n=11)$ was repaired by meatotomy, and partial glans amputation $(n=4)$ was repaired by patching with buccal mucosa (these patients presented too long after circumcision to permit glandular anastomosis). Urethral fistulae $(n=5)$ were repaired by simple closure, and acquired distal hypospadias $(n=3)$ was repaired by the Mathieu procedure in one patient and by tubularized incised plate urethroplasty in the others.

Most complications were caused by poor technique. The classical circumcision procedure has a low complication rate but is difficult to learn, and takes longer to perform than circumcision by clamping and use of a guillotine-like incision; in the hands of inexperienced personnel, however, the latter technique can cause glandular, urethral and corporal injuries.

Original article Ceylan K et al. (2007) Severe complications of circumcision: an analysis of 48 cases. J Pediatr Urol 3: 32-35

\section{Survivors of testicular cancer have an increased risk of second malignancy}

Several studies have indicated that survivors of testicular cancer are at an increased risk of second malignancies because of their exposure to radiotherapy or chemotherapy. In an international collaborative study, Richiardi et al. examined the risk of second malignancies in 29,511 survivors of testicular cancer documented in 13 population-based cancer registries. Standardized incidence ratios (SIRs) for second malignancies were calculated by comparing the number of second malignancies observed in the study population with the expected number obtained from population-specific incidence rates matched for sex, age and year of diagnosis. During 312,216 person-years of follow-up, 1,811 second malignancies were identified.

Following testicular cancer, the SIR for second malignancy was 1.65. Notably, the SIRs for cancer of the stomach, gallbladder and bile ducts, pancreas, bladder, kidney and thyroid were each $\geq 2$, and significantly increased risks were identified for 15 cancer sites altogether. 